\begin{tabular}{|c|c|c|c|c|}
\hline \multicolumn{4}{|c|}{ НАУЧНО-ТЕХНИЧЕСКИЙ ВЕСТНИК ИНФОРМАЦИОННЫХ ТЕХНОЛОГИЙ, МЕХАНИКИ И ОПТИКИ } & \multirow[b]{2}{*}{ 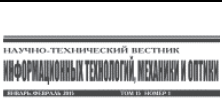 } \\
\hline : & $\begin{array}{cc}\text { март-апрель } 2015 & \text { Том } 15 \text { № } 2 \\
\text { SCIENTIFIC AND TECHNICAL JOURNAL O }\end{array}$ & $\begin{array}{l}\text { ISSN 2226-1494 } \\
\text { NFORMATION TEC }\end{array}$ & $\begin{array}{l}\text { http://ntv.ifmo.ru/ } \\
\text { IECHANICS AND OPTICS }\end{array}$ & \\
\hline УНИВЕРСИТЕТ ИТМО & March-April 2015 & ISSN 2226-1494 & http://ntv.ifmo.ru/en & \\
\hline
\end{tabular}

\author{
УДК 004.042 \\ МОНИТОРИНГ ПОТРЕБЛЕНИЯ ЭНЕРГИИ В УМНЫХ СЕТЯХ \\ ЭЛЕКТРОСНАБЖЕНИЯ (SMART GRID) НА ОСНОВЕ СЕМАНТИЧЕСКОГО \\ АНАЛИЗА ПОТОКОВЫХ ДАННЫХ \\ М.А Колчин ${ }^{a}$, А. Фенцель ${ }^{\mathrm{b}}$, Д.И. Муромцев ${ }^{a}$, С.О. Попов ${ }^{\mathrm{a}}$, Д.С. Павлов ${ }^{\mathrm{a}}$, Н.В. Климов ${ }^{\mathrm{a}}$, \\ А.А. Андреев ${ }^{a}$, Д.С. Гарайзуев ${ }^{a}$ \\ ${ }^{\text {a } У н и в е р с и т е т ~ И Т М О, ~ С а н к т-П е т е р б у р г, ~ 197101, ~ Р о с с и и ̆ с к а я ~ Ф е д е р а ц и я ~}$ \\ ${ }^{\mathbf{b}}$ Семантический технологический институт, Университет Инсбрука, Инсбрук, 6020, Австрия \\ Адрес для переписки: kolchinmax@gmail.com \\ Информация о статье \\ Поступила в редакцию 11.02 .15 , принята к печати 24.02 .15 \\ doi:10.17586/2226-1494-2015-15-2-285-292 \\ Язык статьи - русский \\ Ссылка для цитирования: Колчин М.А., Фенцель А., Муромцев Д.И., Попов С.О., Павлов Д.С., Климов Н.В., Андреев А.А., Гарайзу- \\ ев Д.С. Мониторинг потребления энергии в умных сетях электроснабжения (Smart grid) на основе семантического анализа потоковых \\ данных // Научно-технический вестник информационных технологий, механики и оптики. 2015. Том 15. № 2. С. 285-292.
}

\title{
Аннотация
}

Постановка проблемы. В настоящий момент задачи повышения энергоэффективности решаются, главным образом, за счет создания более экономичных устройств и приборов, использования нетрадиционных источников энергии, применением специального дополнительного оборудования для контроля над энергопотреблением и другими технологическими способами. Все эти решения достаточно дорогостоящи и зачастую экономически трудноокупаемы. В то же время вопросы автоматизированного интегрального анализа существующих данных контрольно-измерительного оборудования изучены мало. Но именно эти данные содержат всю необходимую информацию для поиска узких мест и сбоев в работе оборудования, приводящих к росту энергопотребления.

Методы. Рассмотрены методы построения веб-сервисов для мониторинга текущего состояния электрической сети, используя CQELS для интеграции статических и потоковых данных интеллектуальных счетчиков. В качестве основного способа представления данных используется модель данных RDF.

Результаты. Предложена архитектура системы мониторинга энергопотребления на основе семантического анализа потоковых данных Smart grid. Для построения информационной модели предметной области разработана онтология, описывающая данные измерений и возможные ситуации для отслеживания системой с помощью семантических запросов. Показан пример работы системы и описание интерфейсов визуализации потоковых данных и журнала сообщений.

Практическая значимость. Промышленное внедрение предложенного подхода позволит добиться значимого повышения энергоэффективности за счет интегрального анализа данных умных счетчиков на существующей инфраструктуре контрольно-измерительного оборудования. Дополнительным эффектом является возможность построения гибкой системы мониторинга интеллектуальных электрических сетей и визуализации их состояний за счет применения онтологического подхода к моделированию предметной области.

Ключевые слова: онтологии, RDF, семантический анализ потоковых данных, интеллектуальный счетчик, интеграция данных, визуализация, Smart grid.

Благодарности. Работа выполнена при государственной финансовой поддержке ведущих университетов Российской Федерации (субсидия 074-U01).

\section{ENERGY CONSUMPTION MONITORING OF SMART GRID BASED ON SEMANTIC STREAM DATA ANALYSIS}

M.A. Kolchin ${ }^{a}$, A. Fensel ${ }^{b}$, D.I. Mouromtseva , S.O. Popova , D.S. Pavlov ${ }^{a}$, N.V. Klimova A.A. Andreev ${ }^{a}$, D.S. Garayzuev ${ }^{a}$

a ITMO University, Saint Petersburg, 197101, Russian Federation

b Semantic Technology Institute, University of Innsbruck, Innsbruck, 6020, Austria

Corresponding author: kolchinmax@gmail.com

Article info

Received 11.02.15, accepted 24.02.15

doi:10.17586/2226-1494-2015-15-2-285-292

Article in Russian

For citation: Kolchin M.A., Fensel A., Mouromtsev D.I., Popov S.O., Pavlov D.S., Klimov N.V., Andreev A.A., Garayzuev D.S. Energy consumption monitoring of Smart grid based on semantic stream data analysis. Scientific and Technical Journal of Information Technologies, Mechanics and Optics, 2015, vol.15, no. 2, pp. 285-292. (in Russian) 


\begin{abstract}
.
Problem statement. Currently, the task of improving energy efficiency are addressed mainly through the creation of more efficient devices and appliances, the use of alternative energy sources, application of special additional equipment for power consumption control and other technological methods. All these solutions are quite expensive and often economically difficult to payback. At the same time, the issues of automated integrated analysis of existing data measuring equipment have been poorly known. But just these data contain all the necessary information for finding bottlenecks and failures in the equipment, leading to increased energy consumption.
\end{abstract}

Methods. Methods of web services creation are considered for current state monitoring of electrical networks using CQELS for static and streaming data integration of smart meters. RDF data model is used as the main way of data representation.

Results. The architecture of the energy monitoring system (Smart grid) based on semantic analysis of the streaming data is proposed. Ontology has been worked out, aimed at information domain model creation, which describes the measurement data and the possible situations for tracking by the system using semantic queries. An example of system operation is shown, and description of the visualization interfaces for streaming data and log of messages is given.

Practical relevance. Industrial application of the proposed approach will give the possibility to achieve significant energy efficiency through integrated analysis of smart meters data based on existing infrastructure of test and measurement equipment. An additional effect lies in the ability to create flexible Smart grid monitoring system and visualization of their states by an ontological approach to the domain modeling.

Keywords: ontologies, RDF, stream data semantic analysis, smart meter, data integration, visualization, Smart grid.

Acknowledgements. This work was partially financially supported by the Government of the Russian Federation (Grant 074U01).

\title{
Введение
}

Энергоэффективность к настоящему времени превратилась в актуальную и требующую незамедлительного решения проблему. Согласно отчету ${ }^{1}$, подготовленному Всемирным банком в сотрудничестве с Центром по эффективному использованию энергии (ЦЭНЭФ), Россия может сэкономить до $45 \%$ своего полного потребления первичной энергии и уменьшить эмиссию $\mathrm{CO}_{2}$ до 20\% к 2030 г. по сравнению к уровню 1990 г. С ростом интереса к энергоэффективности растут и соответствующие рынки по автоматизации зданий, умные дома и т.п. Эти факторы ведут к появлению огромного количества данных и следующей за ними необходимости в их управлении и анализе.

С технической стороны, так как интеграция Интернета и «физической реальности» (например, как в Интернете вещей) растет, аналитические и сенсорные системы и сервисы проявляют тенденцию к использованию моделей представления знаний с общими и широко известными структурами для улучшения интероперабельности. Эти данные могут служить основой для разработки различных новых сервисов и услуг. На данный момент эти данные, как правило, собираются, обрабатываются и анализируются вручную и по отдельности каждой компанией, в то время как автоматизация, оптимизация и обмен не только могли бы иметь место, но также имели бы огромное значение для такого большого и важного рынка, как рынок энергоэффективности. Будущее умных сетей электроснабжения (Smart grid) - в постепенном переходе от централизованной модели сети к новой децентрализованной модели с огромным количеством распределенного оборудования [1]. В этом случае потребители и предприятия будут использовать генерирующее оборудование небольшой мощности. Системы управления такими сетями будут получать доступ к данным по энергоснабжению и энергопотреблению в реальном времени посредством публичной, взаимосвязанной и взаимодействующей инфраструктуры, а затем унифицированным способом распределять электроэнергию из различных источников в предприятия и дома. В связи с этим основой Smart grid будет значительное количество интеллектуальных счетчиков (Smart meters) и сенсоров для более точного определения текущего и необходимого энергопотребления и последующего рационального перераспределения потребления и хранения энергии. Таким образом, упростится подключение новых возобновляемых источников энергии, появится возможность снижать негативное влияние пиковых нагрузок на сеть.

В настоящей работе описано применение семантического анализа потоковых данных для организации мониторинга энергопотребления распределенных интеллектуальных систем унифицированного сбора и анализа данных из гетерогенных источников, как статических (например, базы данных) так и динамических (например, сенсоры). Ранее данный подход был использован в таких областях, как управление инфраструктурой облачных вычислений [2], анализ социальных сетей $[3,4]$, мониторинг состояния окружающей среды [5-7], мониторинг движения транспорта [8], обнаружение событий в контексте умного города $[9,10]$ и др.

Дано описание архитектуры программной системы, приведено описание использованных верхнеуровневых онтологий, а также описана онтология интеллектуальных электрических счетчиков. Представлены примеры событий предметной области, которые система должна обнаруживать, показан пользовательский интерфейс системы.

\footnotetext{
1 Энергоэффективность в России: скрытый резерв, http://www.cenef.ru/file/FINAL_EE_report_rus.pdf
} 


\section{Архитектура системы мониторинга энергопотребления}

Семантические веб-технологии уже доказали $[11,12]$, что они могут стать инструментом для решения проблем синтаксической и семантической интеграции данных в гетерогенных средах и в различных форматах. Resource Description Framework (RDF) и Ontology Web Language (OWL) обеспечивают общую модель и словарь для такого типа данных, а язык SPARQL и механизмы логического вывода позволяют динамически формировать специфическую и полностью новую информацию на основе интегрированного массива данных. Более того, технологии обработки потоковых данных RDF [13] позволяют объединять формализованные знания (онтологии) и анализ потоковых данных вместе.

Этот подход может быть применен и к данным так называемых интеллектуальных счетчиков, осуществляющих первичную обработку результатов измерений и передачу информации на сервер по стандартным протоколам. Это делает возможным построение системы мониторинга на существующей инфраструктуре без существенных изменений в конфигурации оборудования за счет интеграции статических и потоковых данных счетчиков, объединенных в сеть, для автоматического реагирования на аварийные ситуации и нештатные события.

Архитектура системы показана на рис. 1. Потоковые данные (текущие показания), поступающие от интеллектуальных счетчиков, преобразуются в модель данных RDF и образуют массив упорядоченных во времени RDF-триплов, который называется RDF-потоком. Для публикации RDF-потоков используется шина сообщений, работающая по протоколу Advanced Messaging Queuing Protocol (AMQP) ${ }^{1}$. Каждый RDF-поток имеет уникальный идентификатор в виде Uniform Resource Identifier (URI), по которому с помощью протокола AMQP можно получать данные в режиме реального времени. Пример уникального идентификатора RDF-потока:

amqp://example.com:10000/vhost?exchangeName=exchange\&routingKey=mercury230_13534128.

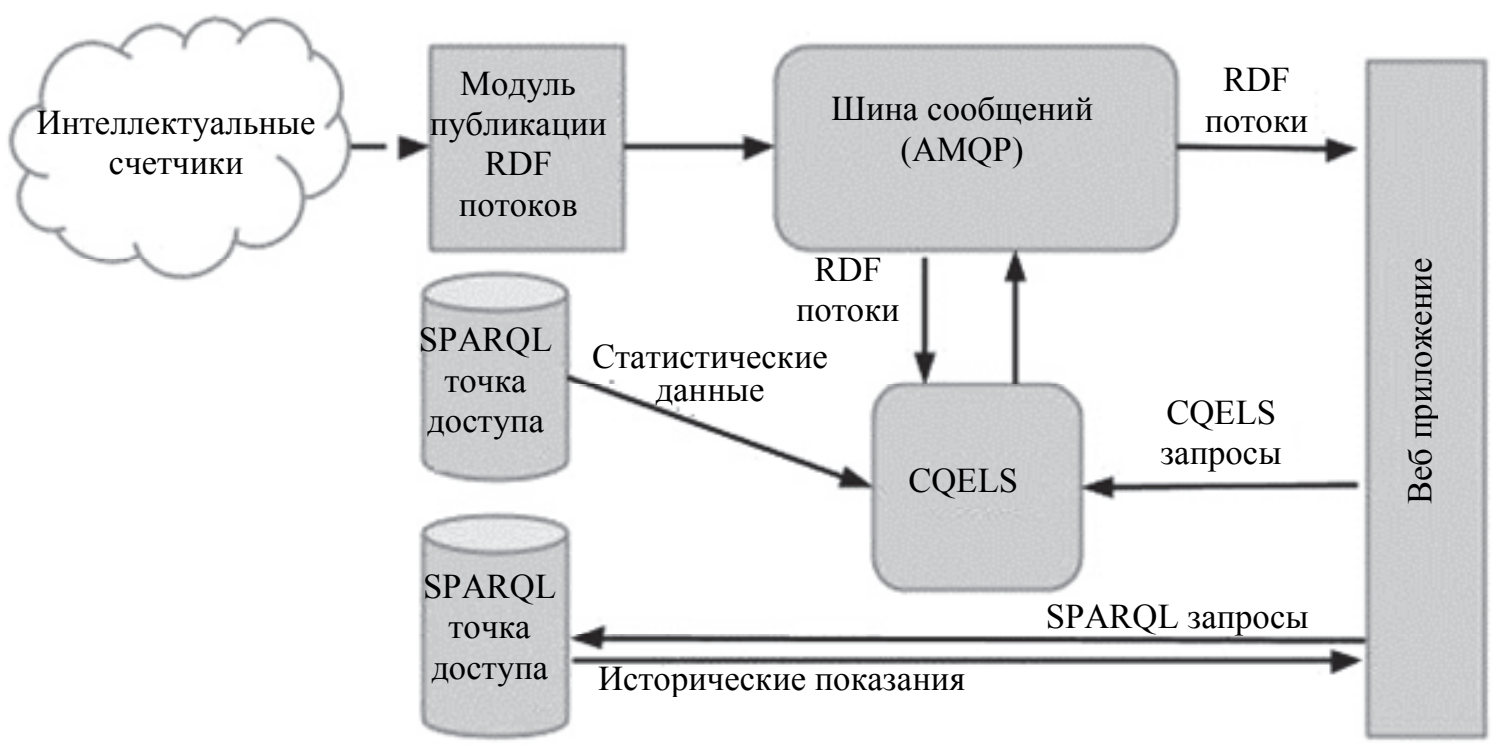

Рис. 1. Архитектура системы: приложение, интеллектуальные счетчики, SPARQL-точки доступа и RDF-потоки

Статические RDF-данные, получаемые от SPARQL-точки доступа, представляют такую информацию об интеллектуальном счетчике, как тип, серийный номер, место установки, точность, дальность действия и т.д. Интеграция статических и потоковых источников RDF-данных осуществляется модулем Соntinuous Query Evaluation over Linked Stream (CQELS) с помощью SPARQL-подобного языка запросов. Описание системы CQELS и языка запросов к статическим и потоковым RDF-данным представлено в работе [14].

B системе используются две SPARQL-точки доступа для хранения информации об интеллектуальных счетчиках и хранения исторических показаний, которые позже используются для визуализации. Необходимость в наличии двух отдельных хранилищ связана с различием требований. Например, новые исторические показания добавляются в хранилище регулярно с определенной частотой, что увеличивает время доступа к данным на чтение, а данные о счетчиках меняются редко, но к ним осуществляется регулярный доступ на чтение.

Разработанное авторами веб-приложение [15] позволяет визуализировать текущие и исторические показания интеллектуальных счетчиков в виде графиков в режиме реального времени и регистрировать

\footnotetext{
${ }^{1}$ Advanced Messaging Queuing Protocol, http://en.wikipedia.org/wiki/Advanced_Message_Queuing_Protocol
} 
CQELS-запросы, которые необходимы для идентификации нештатных или аварийных ситуации. Например, простейшей формой такой ситуации может быть появление данных о слишком высоких значениях напряжения, измеренного, по крайней мере, в одном из подключенных к системе мониторинга интеллектуальных счетчиков.

Результатом запроса является набор RDF-триплов, описывающих сообщение о нештатной или аварийной ситуации с помощью верхнеуровневых и предметных онтологий. Пример запроса и сообщения приводятся в следующих разделах.

\section{Описание онтологической модели}

Для представления показаний интеллектуальных счетчиков и сообщений о нештатных или аварийных ситуациях используются несколько верхнеуровневых и предметных онтологий:

- верхнеуровневая онтология сети семантических сенсоров SSN (Semantic Sensor Networks Ontology) ${ }^{1}$;

- предметная онтология электрических счетчиков EM (ElectricMeter Ontology) ${ }^{2}$, являющаяся расширением SSN;

- верхнеуровневая онтология событий DUL (DOLCE+DnS Ultralite ontology) ${ }^{3}$.

На рис. 2 показан фрагмент данных об интеллектуальном счетчике и его показаниях, аннотированных с помощью перечисленных выше онтологий.

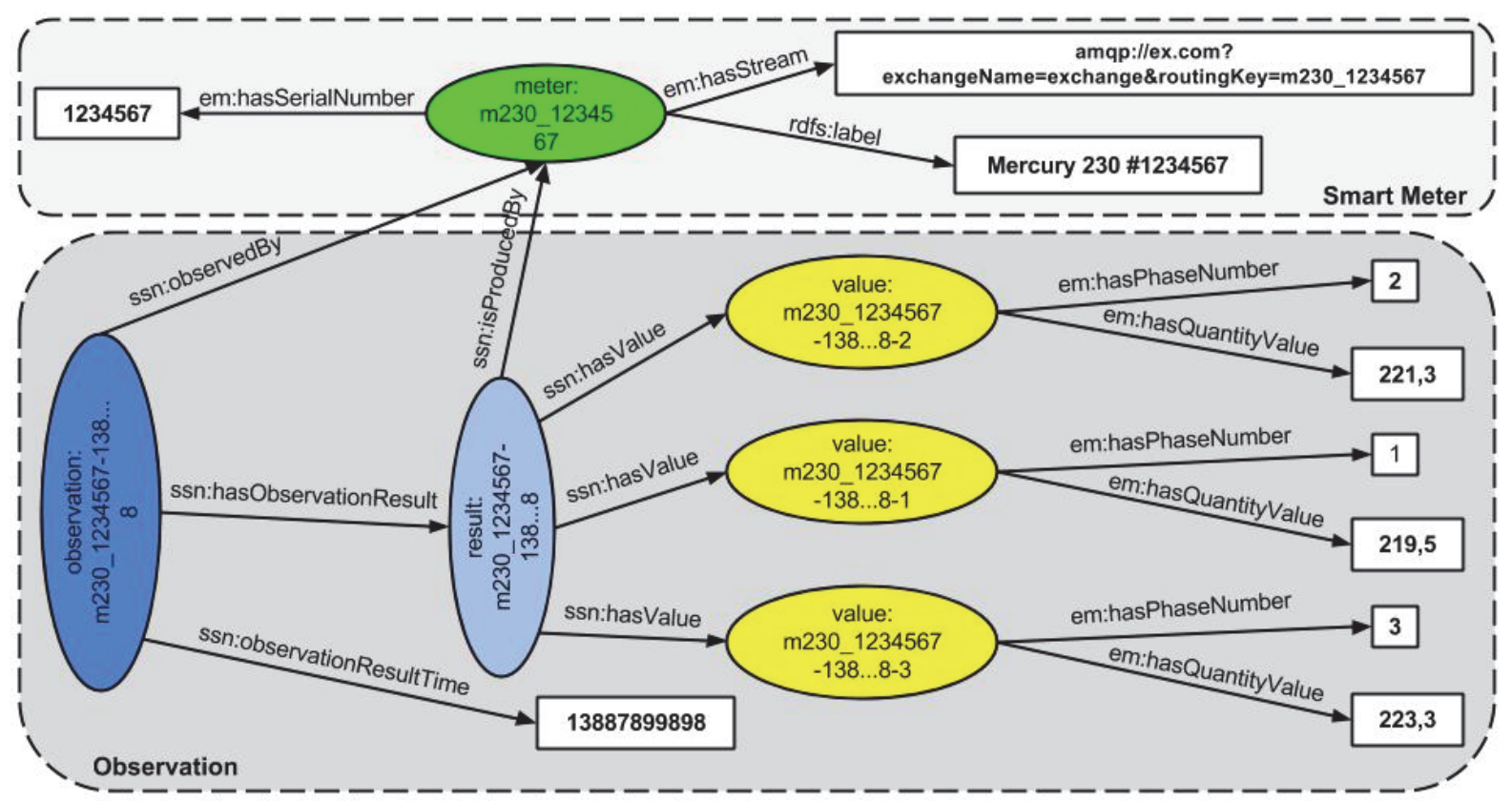

Рис. 2. Онтология интеллектуального счетчика и его показаний

Онтология ЕМ расширяет несколько классов и свойств онтологии SSN, например:

- em:ElectricityFeature является подклассом ssn:FeatureOfInterest для описания свойства, которое данный сенсор наблюдает. В данном случае это электричество;

- em:PolyphaseVoltageObservation является подклассом ssn:Observation для описания показания сенсора;

- em:hasStream - объектное свойство для представления уникального идентификатора RDF-потока;

- em:hasPhaseNumber - строковое свойство для представления номера электрической фазы.

В общей сложности онтология ЕМ включает в себя 2 объектных свойства, 6 строковых свойств, 22 класса и 3 экземпляра.

\section{Пример семантического запроса обработки нештатных ситуаций}

Семантические запросы на основе языка запросов CQELS, описанные в работе [14], влияют как на аспекты онтологического моделирования данных, так и на обработку потоковых данных и связанных статических данных в рамках одной теоретической основы, которая соответственно определяет два типа источников данных - RDF-потоки и RDF-триплы, а также классы операторов для обработки этих типов источников данных. Операторы, используемые в запросе, организованы в потоке данных в соответствии с определенной семантикой запросов, определение которой задано в онтологической модели. Основным

\footnotetext{
${ }^{1}$ Semantic Sensor Network Ontology, http://purl.oclc.org/NET/ssnx/ssn\#

${ }^{2}$ ElectricMeter Ontology, http://purl.org/NET/ssnext/electricmeters\#

${ }^{3}$ DOLCE+DnS Ultralite ontology, http://www.loa-cnr.it/ontologies/DUL.owl\#
} 
классом операторов обработки потоковых данных являются оконные операторы. Эти операторы извлекают RDF-триплы из RDF-потока или набора RDF-данных, которые соответствуют заданному шаблону, и действительны в течение определенного временного интервала (окна). Также в запросах могут быть использованы такие операторы, как реляционный оператор, который схож с реляционным оператором в реляционных базах данных, а также потоковый оператор, который позволяет создавать новые RDFтриплы на основе набора результатов запроса.

Ниже показан пример запроса, формирующего сообщение каждый раз, когда значение напряжения в любой из фаз электрической сети, измеренных одним из интеллектуальных счетчиков, превышает $220 \mathrm{~B}+10 \%$. Все оповещения отправляются в предопределенных RDF-форматах и используются вебприложением для визуализации.

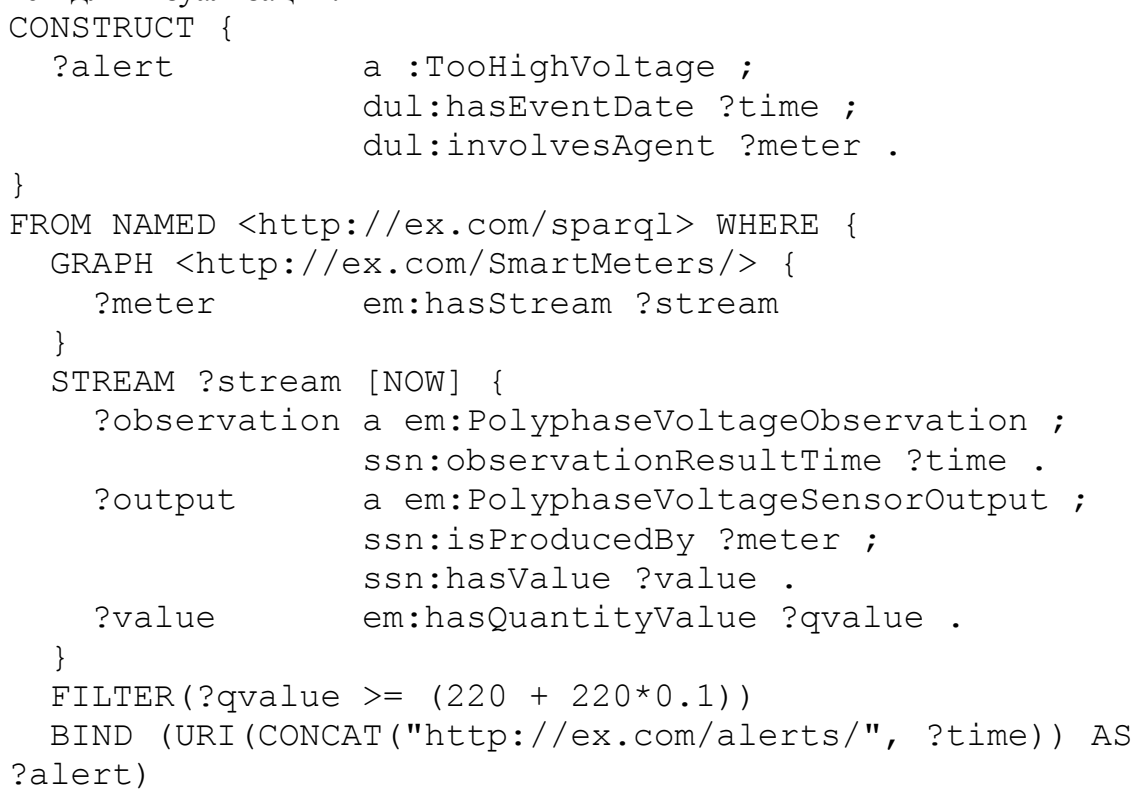

Представленный выше запрос состоит из пяти частей. Выражение CONSTRUCT является реализацией потокового оператора, который создает новые RDF-триплы. В данном случае при появлении новых результатов запроса создаются три новых RDF-трипла, которые описывают событие предметной области. CONSTRUCT \{

?alert a :TooHighVoltage ;

dul:hasEventDate ?time ;

dul: involvesAgent ?meter.

\}

Выражения FROM NAMED и GRAPH являются подзапросом, который отправляется в удаленную базу данных для извлечения соответствующих RDF-триплов.

FROM NAMED <http://ex.com/sparql> WHERE

GRAPH <http://ex.com/SmartMeters/> \{

?meter em:hasstream ?stream

\}

$\cdots$

\}

В данном случае запрашивается список уникальных идентификаторов потока для каждого умного счетчика.

Далее выражение STREAM <..> [NOW] является реализацией оконного оператора, который извлекает описанный RDF-шаблон из текущего временного окна в рамках RDF-потока умного счетчика.

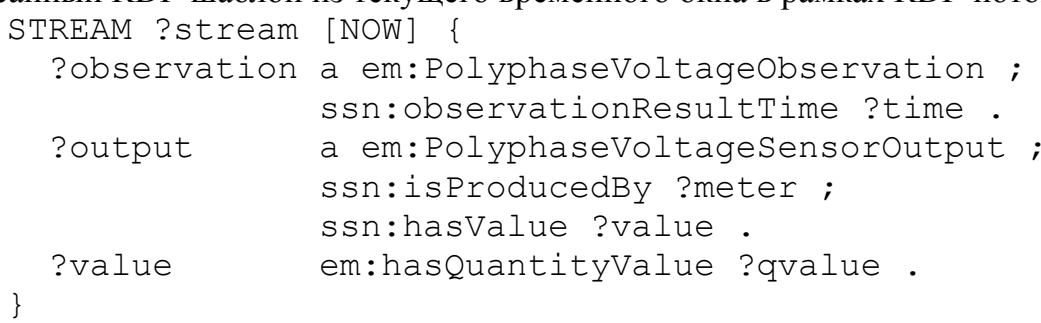

Следующая часть отвечает за фильтрацию результатов запроса по определенному значению, в данном случае по уровню напряжения в сети. 
FILTER (?qvalue $>=(220+220 * 0.1)$ )

Последняя часть запроса реализует механизм создания уникального идентификатора для события предметной области:

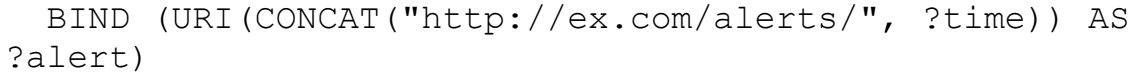

В более сложном случае максимально возможная величина напряжения для различных интеллектуальных счетчиков может изменяться непосредственно в онтологической модели, и вместо создания отдельного оповещения для каждого типа счетчиков эта величина может быть получена из SPARQLточки доступа отдельным дополнительным запросом, что делает данный подход адаптивным к изменениям параметров мониторинга сети.

\section{Пример работы веб-приложения}

Как уже было отмечено выше, для визуализации семантической обработки данных разработано вебприложение. Исходный код опубликован под открытой лицензией: https:/github.com/ailabitmo/DAAFSE.

На рис. 3 приведен интерфейс вывода текущих и исторических потоковых данных показаний интеллектуальных счетчиков в заданный промежуток времени. Информация о счетчике предоставляется SPARQL-точкой доступа из RDF-хранилища статических данных.

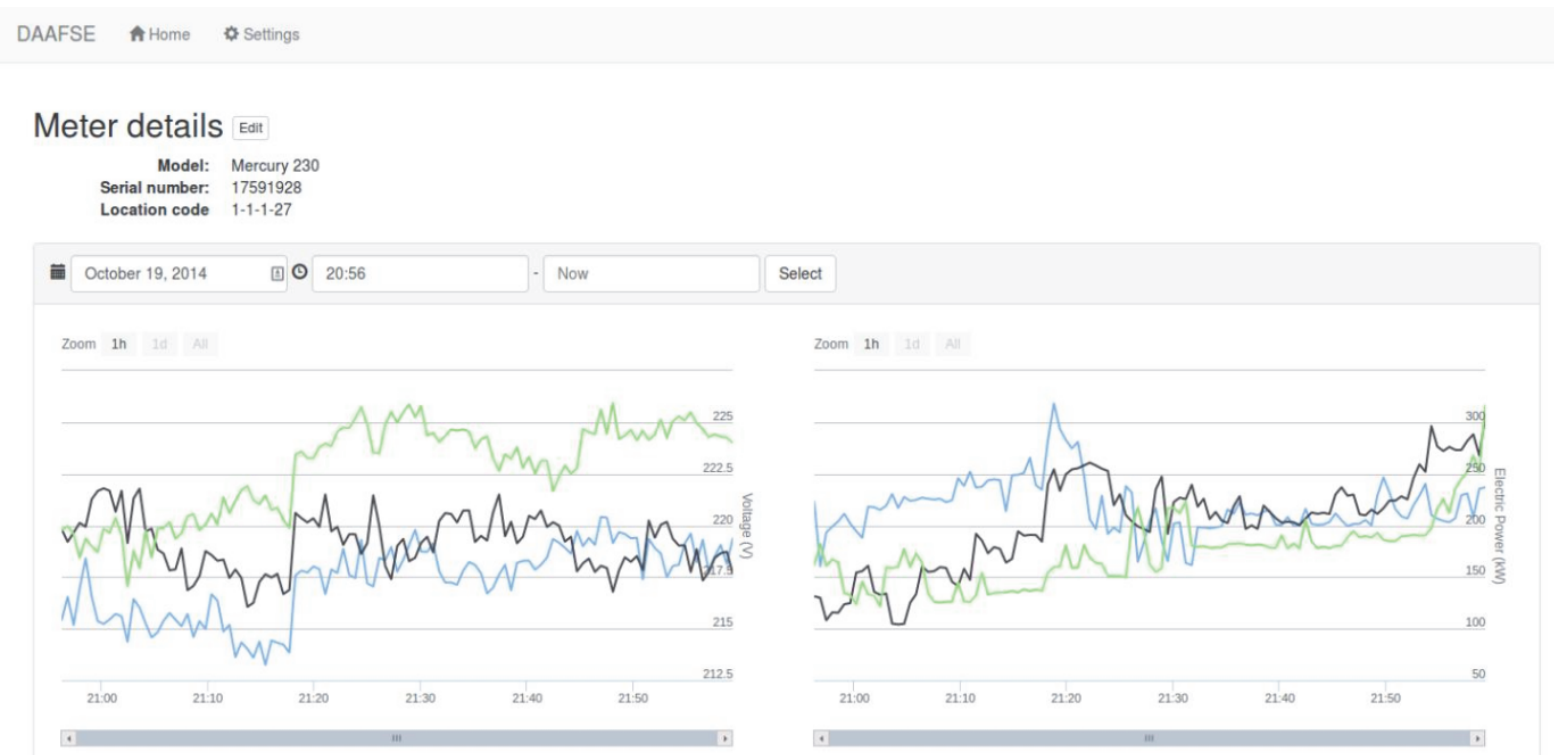

Рис. 3. Интерфейс вывода текущих и исторических показаний: слева расположен график напряжения, а справа - график мощности

По мере формирования сообщений при обработке SPARQL-запросов, аналогичных приведенным в следующем разделе, выполняется их запись в системный журнал (рис. 4). Каждое сообщение содержит в себе информацию о событии и ссылках на интеллектуальный счетчик. При необходимости эта информация может быть использована для выполнения дополнительного SPARQL для получения детальной информации о событии.

\section{Заключение}

В настоящей работе приведена архитектура и примеры работы системы мониторинга потребления энергии в умных сетях электроснабжения (Smart grid) на основе семантического анализа потоковых данных. Архитектура позволяет работать как со статическими данными о параметрах оборудования (интеллектуальных счетчиках), так и с потоковыми данными показаний. Для построения информационной модели предметной области разработана онтология, описывающая данные измерений и возможные ситуации для отслеживания системой с помощью семантических запросов. Приведены примеры семантических запросов для определения нештатных ситуаций. Наконец, показан пример работы системы и описание интерфейсов визуализации потоковых данных и журнала сообщений. Система проходила опытную эксплуатацию на предприятии «Ленполиграфмаш», где показала высокую эффективность при минимальных затратах на ее внедрение. 


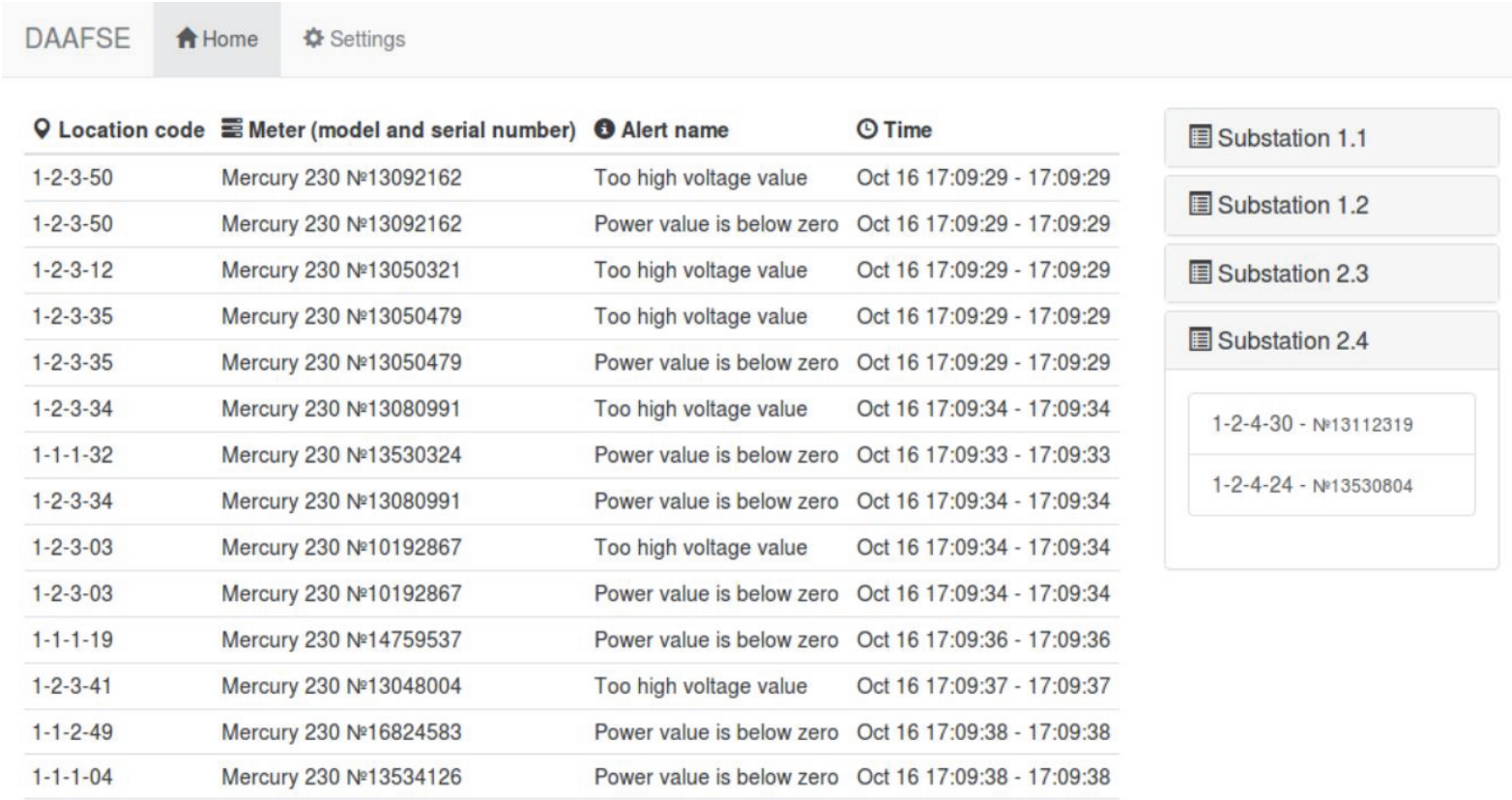

Рис. 4. Журнал сообщений о нештатных и аварийных ситуациях

\section{Литература}

1. Brown R.E. Impact of smart grid on distribution system design // Proc. IEEE Power and Energy Society General Meeting: Conversion and Delivery of Electrical Energy in $21^{\text {th }}$ Century. Pittsburgh, USA, 2008. Art. 4596843. doi: 10.1109/PES.2008.4596843

2. Dautov R., Paraskakis I., Stannett M. Towards a framework for monitoring cloud application platforms as sensor networks // Cluster Computing. 2014. V. 17. P. 1203-1213. doi: 10.1007/s10586-014-0389-5

3. Barbieri D., Braga D., Ceri S., Della Valle E., Huang Y., Tresp V., Rettinger A., Wermser H. Deductive and inductive stream reasoning for semantic social media analytics // IEEE Intelligent Systems. 2010. V. 25. N 6. P. 32-41. doi: 10.1109/MIS.2010.142

4. Celino I., Dell Agilo D., Della Valle E., Huang Y., Lee T., Kim S., Tresp V. Towards BOTTARI: using stream reasoning to make sense of location-based micro-posts // Lecture Notes in Computer Science. 2012. V. 7117. P. 80-87. doi: 10.1007/978-3-642-25953-1 7

5. Wetz P., Trinh T.D., Do B.L., Anjomshoaa A., Kiesling E., Tjoa A.M. Towards an environmental information system for semantic stream data // Proc. $28^{\text {th }}$ Conf. on Environmental Informatics - Informatics for Environmental Protection, Sustainable Development and Risk Management. Oldenburg, Germany, 2014. P. 637-644.

6. Taylor K., Leidinger L. Ontology-driven complex event processing in heterogeneous sensor networks // Lecture Notes in Computer Science. 2011. V. 6643. P. 285-299. doi: 10.1007/978-3-642-21064-8_20

7. Gray A., Sadler J., Kit O., Kyzirakos K., Karpathiotakis M., Calbimonte J.-P., Page K. et. al. A semantic sensor web for environmental decision support applications // Sensors. 2011. V. 11. N 9. P. 8855-8887. doi: $10.3390 / \mathrm{s} 110908855$

8. Heintz F., Kvarnstrom J., Doherty P. Bridging the sense-reasoning gap: DyKnow - stream-based middleware for knowledge processing // Advanced Engineering Informatics. 2010. V. 24. N 1. P. 14-26. doi: 10.1016/j.aei.2009.08.007

9. Banerjee S., Mukherjee D., Misra P. 'What affects me?' A smart public alert system based on stream reasoning // Proc. $7^{\text {th }}$ Int. Conf. on Ubiquitous Information Management and Communication (ICUIMC 2013). Kota Kinabalu, Malaysia, 2013. Art. 22. doi: 10.1145/2448556.2448578

10. Ruta M., Scioscia F., Di Sciascio E., Rotondi D., Piccione S. Semantic-based knowledge dissemination and extraction in smart environments // Proc. $27^{\text {th }}$ Int. Conf. on Advanced Information Networking and Applications Workshops (WAINA 2013). Barcelona, Spain, 2013. P. 1289-1294. doi: 10.1109/WAINA.2013.249

11. Tallevi-Diotallevi S., Kotoulas S., Foschini L., Lecue F., Corradi A. Real-time urban monitoring in Dublin using semantic and stream technologies // Lecture Notes in Computer Science. 2013. V. 8219. P. 178-194. doi: 10.1007/978-3-642-41338-4_12

12. Balduini M., Della Valle E., Dell Aglio D., Tsytsarau M., Palpanas T., Confalonieri C. Social listening of city scale events using the streaming linked data framework // Lecture Notes in Computer Science. 2013. V. 8219. P. 1-16. doi: 10.1007/978-3-642-41338-4_1

13. Calbimonte J., Corcho O., Gray A. Enabling ontology-based access to streaming data sources // Lecture Notes in Computer Science. 2010. V. 6496. P. 96-111. doi: 10.1007/978-3-642-17746-0_7 
14. Le-Phuoc D., Dao-Tran M., Xavier Parreira J., Hauswirth M. A native and adaptive approach for unified processing of linked streams and linked data // Lecture Notes in Computer Science. 2011. V. 7031. P. 370-388.

15. Kolchin M., Mouromtsev D., Arustamov S. Demonstration: web-based visualisation and monitoring of smart meters using CQELS // Proc. $7^{\text {th }}$ Int. Workshop on Semantic Sensor Network. Trentino, Italy, 2014. P. 1-4.

Колчин Максим Александрович

Фенцель Анна

Муромцев Дмитрий Ильич

Попов Сергей Олегович

Павлов Дмитрий Сергеевич

Климов Николай Васильевич

Андреев Алексей Андреевич

Гарайзуев Даниил Сергеевич

Maxim A. Kolchin

Anna Fensel

Dmitry I. Mouromtsev

Sergei O. Popov

Dmitry S. Pavlov

Nikolai V. Klimov

Alexei A. Andreev

Daniil S. Garayzuev
- аспирант, Университет ИТМО, Санкт-Петербург, 197101, Российская Федерация, kolchinmax@gmail.com

- $\mathrm{PhD}$, старший научный сотрудник, Семантический технологический институт, Университет Инсбрука, Инсбрук, 6020, Австрия, anna.fensel@sti2.at

- $\quad$ кандидат технических наук, доцент, доцент, Университет ИТМО, СанктПетербург, 197101, Российская Федерация, mouromtsev@mail.ifmo.ru

- инженер, Университет ИТМО, Санкт-Петербург, 197101, Российская Федерация, sp@winghouse.ru

- лаборант-исследователь, Университет ИТМО, Санкт-Петербург, 197101, Российская Федерация, dmitry.pavlov@vismart.biz

- студент, Университет ИТМО, Санкт-Петербург, 197101, Российская Федерация, hocico16@gmail.com

- студент, Университет ИТМО, Санкт-Петербург, 197101, Российская Федерация, aandreyev13@gmail.com

- студент, Университет ИТМО, Санкт-Петербург, 197101, Российская Федерация, garayzuev@gmail.com

- $\quad$ postgraduate, ITMO University, Saint Petersburg, 197101, Russian Federation, kolchinmax@gmail.com

- $\quad \mathrm{PhD}$, senior researcher and lecturer, senior researcher and lecturer, Semantic Technology Institute, University of Innsbruck, Innsbruck, 6020, Austria, anna.fensel@sti2.at

- $\mathrm{PhD}$, Associate professor, Associate professor, ITMO University, Saint Petersburg, 197101, Russian Federation, mouromtsev@mail.ifmo.ru

- engineer, ITMO University, Saint Petersburg, 197101, Russian Federation, sp@winghouse.ru

- laboratory researcher, ITMO University, Saint Petersburg, 197101, Russian Federation, dmitry.pavlov@vismart.biz

- $\quad$ student, ITMO University, Saint Petersburg, 197101, Russian Federation, hocico16@gmail.com

- student, ITMO University, Saint Petersburg, 197101, Russian Federation, aandreyev13@gmail.com

- student, ITMO University, Saint Petersburg, 197101, Russian Federation, garayzuev@gmail.com 